\title{
Prevalence of nontuberculous mycobacteria in patients with bronchiectasis: a meta-analysis
}

Haiqing Chu', Lan Zhao', Heping Xiao ${ }^{2}$, Zhemin Zhang ${ }^{1}$, Jinbo Zhang' ${ }^{1}$, Tao Guil, Sugang Gong', Liyun $\mathrm{Xu}^{1}$, Xiwen Sun ${ }^{3}$

\author{
${ }^{1}$ Department of Respiratory Disease, Shanghai Pulmonary Hospital, Tongji University \\ School of Medicine, Shanghai, China \\ 2Department of Tuberculosis, Shanghai Pulmonary Hospital, Tongji University School \\ of Medicine, Shanghai, China \\ ${ }^{3}$ Department of Radiology, Shanghai Pulmonary Hospital, Tongji University School \\ of Medicine, Shanghai, China
}

Submitted: 9 May 2013

Accepted: 24 May 2013

Arch Med Sci 2014; 10, 4: 661-668

DOI: 10.5114/aoms.2014.44857

Copyright $\odot 2014$ Termedia \& Banach

\section{Abstract}

Introduction: Nontuberculous mycobacteria (NTM) have emerged as critical opportunistic pathogens of lung diseases recently. Patients with preexisting bronchiectasis are susceptible to NTM. Nevertheless, patients with preexisting bronchiectasis are susceptible to NTM but the prevalence of NTM pulmonary infection in different species and geographical areas is still not fully understood.

Material and methods: The relevant data of the prevalence of NTM in patients with bronchiectasis were retrieved by searching the main databases such as PubMed, MEDLINE, Cochrane Library, and EMBASE. This meta-analysis was performed using Rev. Man 5.1 and Stata 11.0 software. The collected information of NTM prevalence was chosen as the effect size.

Results: The results of the meta-analysis showed that the overall prevalence of NTM was $9.3 \%$ in patients with bronchiectasis. The further stratification of subgroup analysis indicated that the combined prevalence of NTM was higher in studies whose "sample size" was more than or equal to 100 $(p=0.002)$, in studies in which "time of study" was after or equal to 2002 $(p<0.001)$, in studies in which "participants' geographic location" was Asian $(p<0.001)$ and in studies whose "method of study" was retrospective ( $p=0.002)$ as well, compared with corresponding groups.

Conclusions: Our findings suggested that the prevalence NTM infection is high in patients with bronchiectasis. A larger number of definitive randomized trials are still required to assess this research issue.

Key words: bronchiectasis, nontuberculous mycobacteria, prevalence, meta-analysis.

\section{Introduction}

Bronchiectasis is defined pathologically as abnormal and irreversible dilatation of the bronchi, with the potential to cause devastating illness including chronic productive cough, airway obstruction, shortness of breath and recurrent infections [1]. There is usually an initial event which causes impairment of mucociliary clearance of the bronchial tree. The respiratory tract becomes colonized by bacteria that inhibit ciliary function and promote further lung damage by the host's immune system [2]. Non-

\author{
Corresponding author: \\ Heping Xiao \\ No. 507 Zheng Min Road \\ Shanghai 200433, China \\ Phone: +86-021-65115006 \\ Fax: +86-021-65115006 \\ E-mail: xiaohepingqqq@ \\ hotmail.com \\ Zhemin Zhang \\ No. 507 Zheng Min Road \\ Shanghai 200433, China \\ Phone: +86-021-65115006 \\ Fax: +86 02165115006 \\ E-mail: zhangzheminzzz@ \\ hotmail.com
}


tuberculous mycobacteria (NTM) are ubiquitous environmental organisms that sometimes cause respiratory disease $[3,4]$. Because person-to-person transmission has been less reported, it has been proposed that the environment is the major source of NTM infection [5]. Representatives of many species, such as Mycobacterium avium, $M$. fortuitum and $M$. chelonae, have been recovered from environmental samples (e.g., water and soil) [6]. Moreover, DNA fingerprints have been shown to be identical between patient and environmental isolates [7]. Of the NTM species, mycobacterium avium complex (MAC) is one of the most common pathogens of NTM pulmonary infection [8]. Levin has suggested that the combination of bronchiectasis and parenchymal nodules had an overall sensitivity of $80 \%$ and a specificity of $87 \%$ for positive MAC culture and patients with bronchiectasis are predisposed to infection with NTM [9].

As several studies have reported an increasing incidence of NTM in patients with bronchiectasis, recently [10-12], it is therefore important to investigate whether differences of NTM species and geographical area influence NTM prevalence in patients with bronchiectasis. Environmental habitats occupied by nontuberculous mycobacteria are also shared by humans, and the number of individuals with heightened susceptibility to mycobacterial disease is growing (e.g., through immunosuppression) [13]. It is therefore important to investigate whether a geographical difference is associated with NTM prevalence. To date, no systematic reviews regarding these patients are available.

In the present study, we conducted a systematic review of published findings and used meta-analysis techniques to quantitatively combine results of NTM prevalence in patients with bronchiectasis.

\section{Material and methods}

\section{Source of materials}

This meta-analysis was performed based on the Quality of Reporting of Meta-analyses guidelines [14].

We searched multiple electronic bibliographic databases without language restriction, including PubMed, MEDLINE and EMBASE, Springer, Elsevier Science Direct, Cochrane Library and Google Scholar (literature was published up to December 2012). The key words of "bronchiectasis", "bronchiectasis", "nontuberculous mycobacterium", "NTM", "non-tuberculosis mycobacterial", "mycobacterium", "study" and "trial" were used for searching. Meanwhile, references from retrieved papers were checked for any additional study. We only recruited full-published papers, not any meeting or conference abstracts.

\section{Inclusion and exclusion criteria of studies}

Inclusion criteria of studies mainly comprised: 1) an investigation for patients with bronchiectasis (prospective studies, retrospective studies or cross-sectional studies, etc.); 2) the definition of bronchiectasis; 3 ) the effect size of the prevalence of NTM; 4) sample size $\geq 50$; 5) papers that were published before 2012. In addition, we excluded studies if they only described NTM data unassociated with bronchiectasis, were reduplicated studies or records, or did not calculate the prevalence of NTM in the patients of bronchiectasis.

\section{Search methods}

Two investigators independently searched the electronic databases. An independent PubMed and MEDLINE search was done (by LZ and HX) with the same method. An independent Springer and Elsevier Science Direct search was done (by ZZ and JZ) with the same method. An independent Cochrane Library and Google Scholar search was done (by TG and SG) with the same method. The abstracts were reviewed independently by two investigators (by LZ and ZZ) to determine if they met eligibility criteria for inclusion. References in the studies were reviewed (by HX and JZ) to identify additional studies. Where discrepancies occurred, a third investigator (TG) did additional assessment.

\section{Evaluation of quality and extraction of data}

Evaluation of quality mainly included methods of studies, sample size, and recruitment of respondents. Initial screening was done by reading the document title and abstract, then reading the full text of papers for secondary screening, to determine whether studies were included according to inclusion criteria finally. Two investigators independently completed this course, if there were any discrepancies, by means of discussion in order to reach an agreement.

We developed and modified a data abstraction form after a training exercise for investigators. Data items were study details (e.g., the first author's name, year of study, year of publication, location of participants, method of studies), characteristics of participants (e.g., age, sample size), and the prevalence of NTM in the patients of bronchiectasis. Two investigators (LZ and JZ) extracted the data independently using the standard protocol and the result was reviewed by a third investigator $(\mathrm{HX})$. We contacted the authors of the studies included to obtain further information for data items that needed clarification. Discrepancies were resolved by discussion with our research team or contract with original investigators, who were all sent data extraction sheets with requests 
for correction. We recorded the first author's name, time of study, time of publication, country, geographic location, sample size, age, study method and the prevalence of NTM.

\section{Meta-analysis methods}

The meta-analysis combined the prevalence of NTM in the patients of bronchiectasis, and then studies stratified by sample size, time of study, participants' geographic location and method of study, using subgroup analysis.

The meta-analysis was performed in fixed and random effect models. The point estimates of effect size, the prevalence of NTM, and its $95 \%$ confidence interval $(95 \% \mathrm{Cl})$ were estimated for each study. We assessed the within- and between-study variation or heterogeneity by testing Cochran's Q-statistic [15]. This heterogeneity test assessed the null hypothesis that all studies were evaluating the same effect. A significant $Q$-statistic $(p<0.10)$ indicated heterogeneity across studies, and then the random effect model was used for meta-analysis as well as to take into account the possibility of heterogeneity between studies. Otherwise, the fixed effect model was used. The fixed effect model assumes that all of the studies estimate the same underlying effect and considers only within-study variation. We also quantified the effect of heterogeneity using $R^{2}=100 \% \times(Q-\mathrm{d} f) / Q$ [16]. The $R^{2}$-statistic measures the degree of inconsistency in the studies by calculating what percentage of the total variation across studies results from heterogeneity rather than by chance.

The overall or pooled estimate of effect size (the prevalence of NTM) was obtained using the Mantel and Haenszel method in the fixed effect model [17] and using the DerSimonian and Laird method in the random effect model [18]. The calculation of the pooled effect size in the meta-analysis was performed weighting individual effect sizes by the inverse of their variance. The significance of the pooled effect size was determined by the $Z$-test.

\section{Evaluation of publication bias}

Egger's linear regression test was used to measure the asymmetry of the funnel plot. If there is asymmetry, with smaller studies showing effects that differ systematically from larger studies, the regression line will not run through the origin. The intercept $\alpha$ provides a measure of asymmetry - the larger its deviation from zero, the more pronounced the asymmetry. Funnel plots were used to detect publication bias, but they required a range of studies of varying sizes and subjective judgment, and thus we evaluated publication bias using Egger's linear regression test [19], which measures funnel plot asymmetry on the natural logarithm scale of the effect size.
Analyses were performed using the software Review Manager 5.1 (Cochrane Collaboration, http://ims.cochrane.org/revman) and the STATA software package v. 11.0 (Stata Corporation, College Station, TX, USA). All the $p$ values were two sided. A $p$ value less than 0.05 was considered statistically significant. In order to test the reliability of the results, we also performed the sensitivity analysis after removal of the biggest [20] or smallest [21] sample size of studies or the study with the biggest prevalence of NTM [22] to repeat the meta-analysis.

\section{Results}

\section{Characteristics of eligible studies}

The study selection process is shown in Figure 1. Initially, the electronic searches yielded 6012 items (PubMed: 2057; MEDLINE: 1505; Springer: 968; Elsevier Science Direct: 782; Cochrane Library: 68; Google Scholar: 632), of which 662 studies were removed because of duplication. In addition, 640 of these articles were excluded on the basis of the title and abstract (88 were review articles; 132 not bronchiectasis; 420 did not report NTM prevalence data). Fourteen of the remaining 22 studies were excluded upon a full text search (11 for only NTM data reported but not for bronchiectasis; 3 due to data not available).

Eight eligible studies [20-27] were recruited in the meta-analysis, and the characteristics of included studies are summarized in Table I. Trial participants were aged 13-88 years. Geographic location included Europe, Asia and Australia and

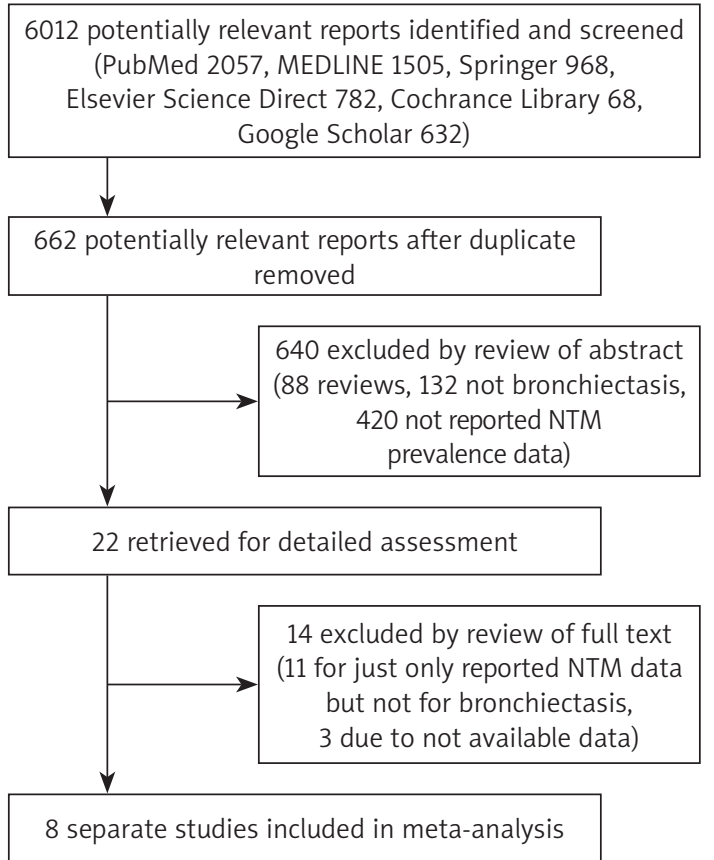

Figure 1. Flow diagram for selecting a study for metaanalysis 


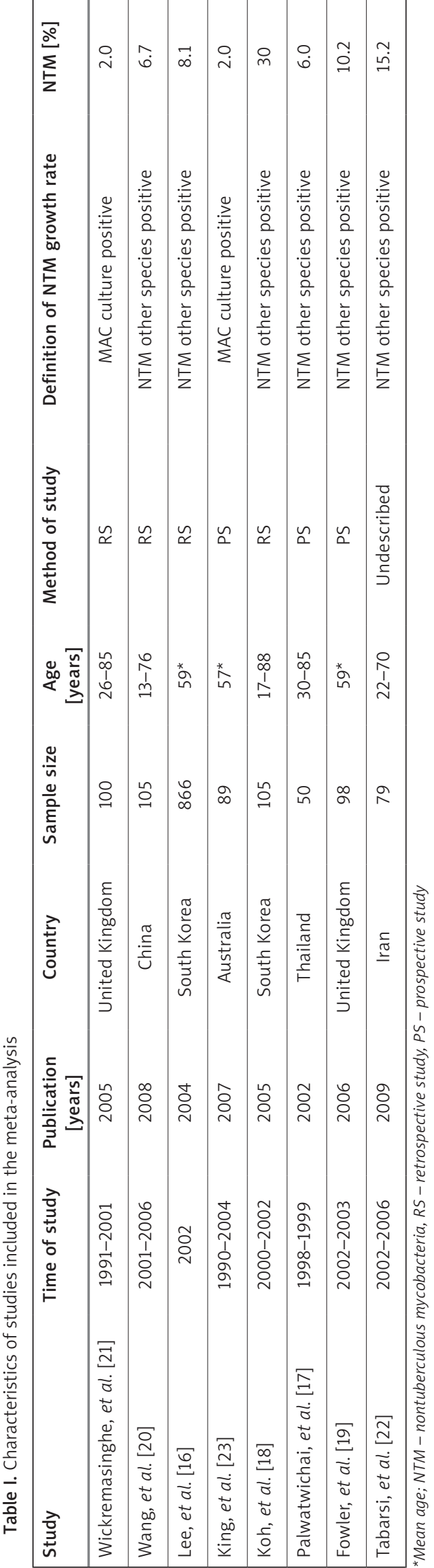

the majority of participants were of east Asian (75.4\%). The prevalence of NTM was detected from $2.0 \%$ to $34.3 \%$ among patients with bronchiectasis. We performed subgroup analysis on the basis of simple size, time of study, participants' geographic location, method of study and different species of NTM. In addition, diagnostic methods for NTM mainly included sputum examination, Haemophilus influenza examination and chest CT scan. NTM growing rate was defined as positive MAC isolates as well as positive sputum cultures for NTM species.

\section{Overall effects}

A total of 8 separate studies, consisting of 1492 bronchiectasis patients, were included in this meta-analysis. The heterogeneity test showed that there were heterogeneities between studies $\left(Q^{2}=\right.$ $64.62, p^{2}=89.2 \%, p<0.01$ ), so we used the random effect model to combine the prevalence of NTM. The overall meta-analysis indicated that the combined prevalence of NTM was $9.3 \%(95 \% \mathrm{Cl}$ 5.0-13.6\%) in patients with bronchiectasis (Figure 2). Publication bias was assessed by inspection of the funnel plot. For NTM prevalence, the distribution of studies was asymmetrical, explaining that publication bias may exist in this meta-analysis (Figure 3). However, the results of Egger's linear regression test did not show there was publication bias in this study $(t=1.27, p>0.05)$ (Table II).

\section{Subgroup analyses for NTM prevalence in bronchiectasis}

We performed a subgroup analysis that was stratified by sample size, time of study, participants' geographic location and method of study. The further stratification of subgroup analysis indicated that the pooled prevalence of NTM was higher when sample size $\geq 100$ compared to sample size < $100(1.1 \%$, 95\% Cl (4.1-18.2\%) vs. $7.7 \%$ $95 \% \mathrm{Cl}(1.8-13.6 \%))$. The analysis of study time indicated that the combined prevalence was lower before 2002 (2.8\%, 95\% Cl (0.4-0.6\%) vs. 9.6\%, $95 \% \mathrm{Cl}(6.2-13.1 \%))$. The geographical difference of NTM illustrated that participants in Asia were more likely to be infected by NTM than those in Europe $(13.1 \%, 95 \% \mathrm{Cl}(6.3-19.9 \%)$ vs. $5.6 \%, 95 \% \mathrm{Cl}$ (2.3-13.6\%)). Furthermore, prevalence of NTM was higher when the method of study was retrospective compared to prospective $(5.5 \%, 95 \% \mathrm{Cl}(0.4-10.7 \%)$ vs. $11.1 \%, 95 \% \mathrm{Cl}(4.1-18.2 \%))$ (Table III).

\section{Subgroup analyses for different species of NTM}

Subgroup analysis of different NTM species is illustrated in Table III. The two most prevalent 


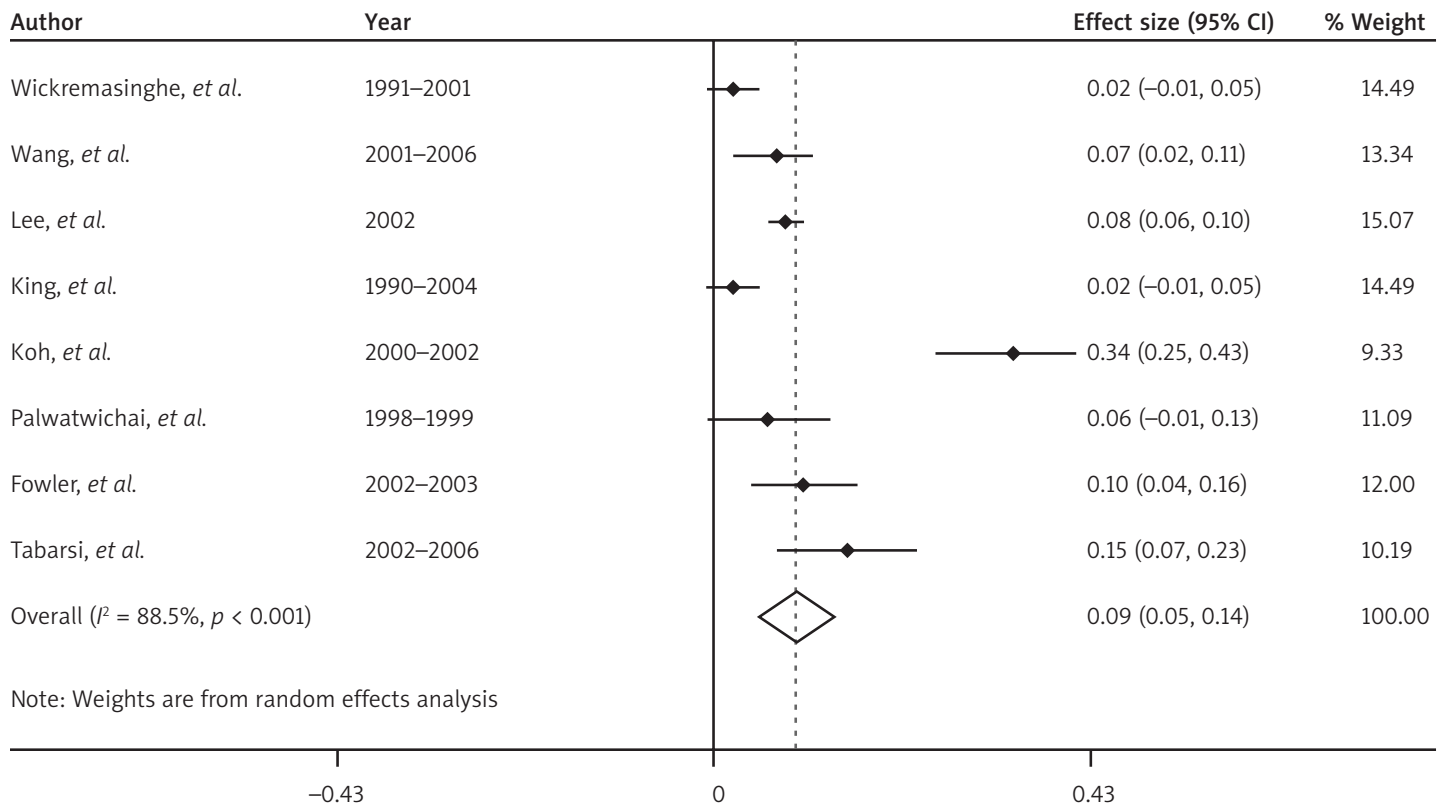

Figure 2. Forest plot for meta-analysis of the prevalence of NTM in patients with bronchiectasis

NTM species were MAC $(25.7,95 \% \mathrm{Cl}(12.6,38.8))$ and $M$. abscessus $(43.2,95 \% \mathrm{Cl}(26.6,60.9)) \mathrm{com}-$ pared to others. Although pooled prevalence of M. chelonae was lower $(3.5,95 \% \mathrm{Cl}(0.9,6.2))$, it has been widely investigated in 5 studies [21, 2326]. In addition, the heterogeneity has been identified in the group of MAC $\left(p<0.001, R^{2}=96.9\right)$.

\section{Evaluation of sensitivity analysis}

The sensitivity analysis showed that the meta-analysis estimates were unchanged after removal of the study that had the biggest [20] or smallest sample size or the highest prevalence of NTM [22].

\section{Discussion}

To date, this is the first meta-analysis investigating the prevalence of NTM in patients with bronchiectasis. Many previous studies have reported the prevalence of NTM in patients with bronchiectasis. However, those studies were limited by small size of samples and weak statistical power. In this study, 8 eligible studies that included 1492 patients with bronchiectasis were collected. The overall meta-analysis showed that the combined prevalence of NTM was 9.3\% (95\% Cl 5.0-13.6\%) in patients with bronchiectasis, indicating that NTM infection was common in patients with bronchiectasis.

The subgroup analysis also showed that the combined prevalence of NTM was higher when the sample size was $\geq 100$, time of study was after 2002, geographic location was in Asia, and method of study was retrospective, compared with prospective groups. The reason that NTM was

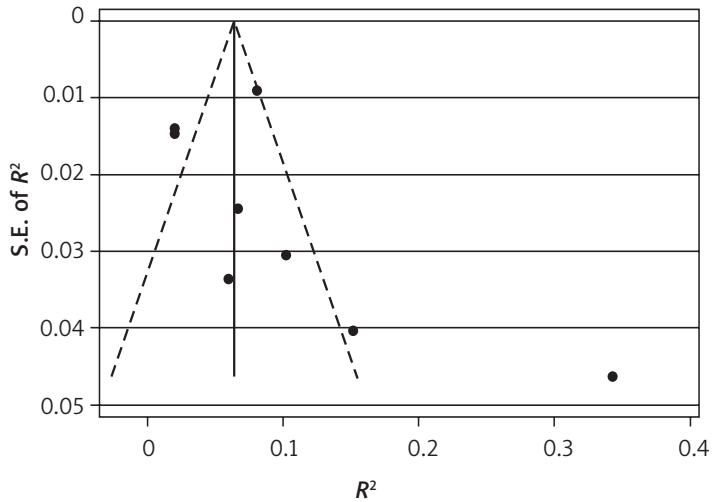

Figure 3. Funnel plot for meta-analysis of the prevalence of NTM in patients with bronchiectasis

more prevalent in the larger sample size $(\geq 100)$ is unclear. A possibility is that substantial random errors may confound the results when the sample size is small. In general, a small sample may have a higher rate of random errors compared to a large sample [28]. Additionally, reasons for the increased reports (research year $\geq 2002$ ) of NTM patients with bronchiectasis may be related to: i) active searching for NTM; ii) improvements in culture technique that reduce bacterial overgrowth; iii) factors that favor transmission such as contaminated air and water supplies; and iv) a more susceptible host reflecting increasing NTM infection in the general population [29]. Furthermore, our finding confirmed that the difference of participants' geographic location is highly associated with the prevalence of NTM. It is speculated that the prevalence of NTM infection is higher in Asia because of inadequate safe water supply, poor sanitation and living conditions. 


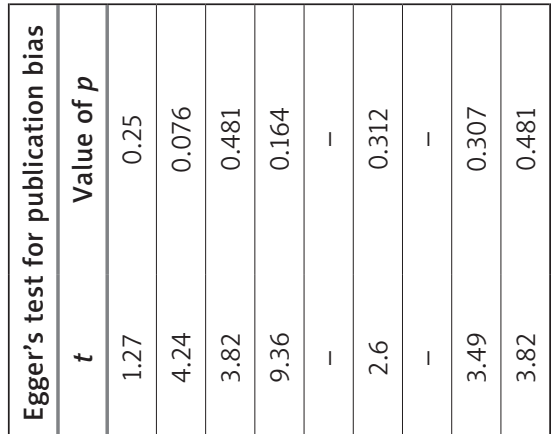

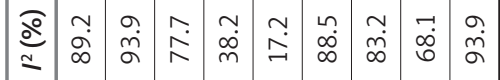
$\frac{3}{3}$

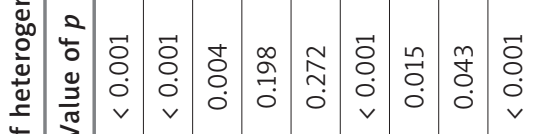

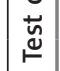

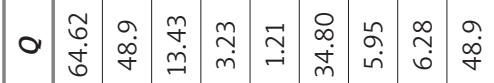

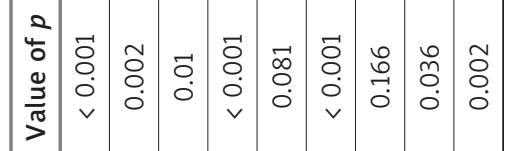

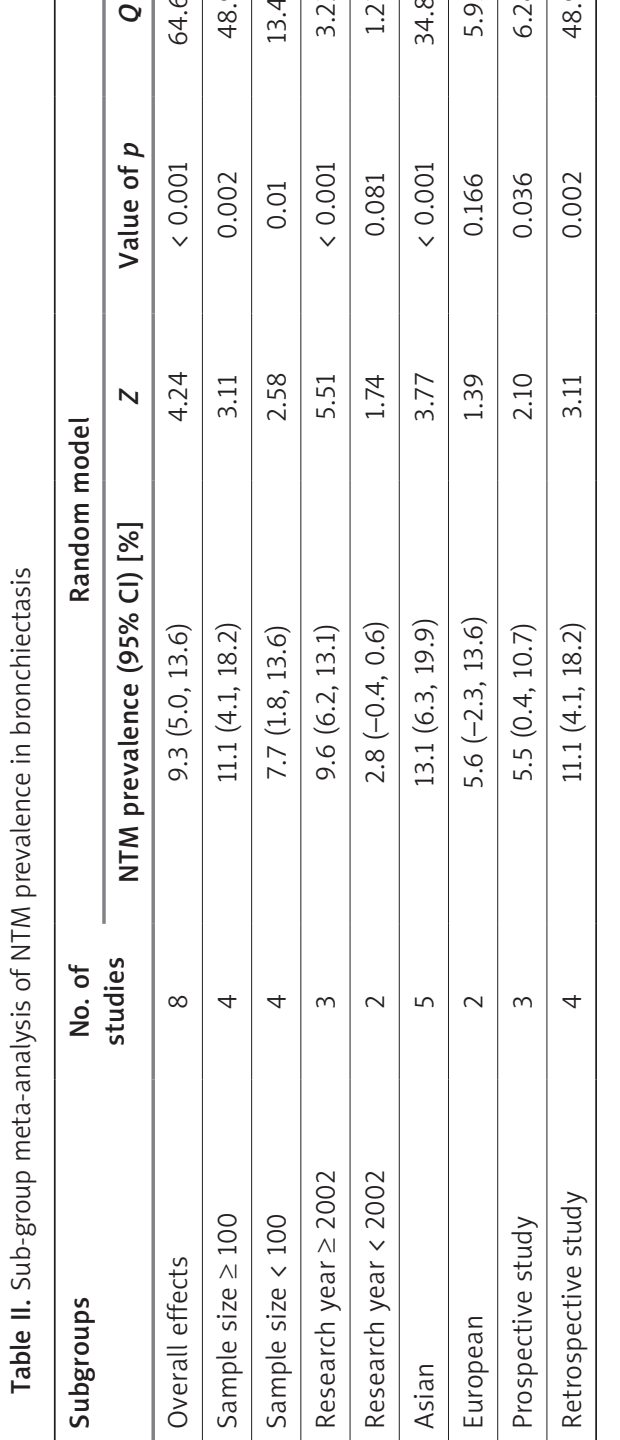

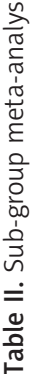

\section{.}

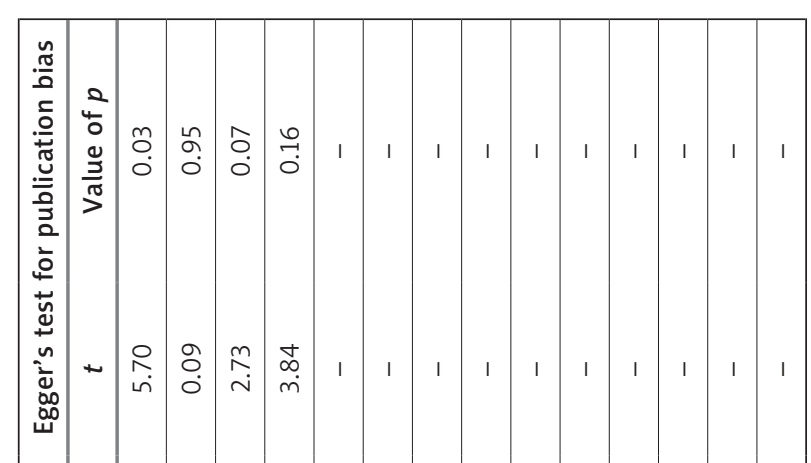

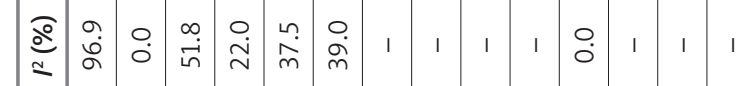
妾

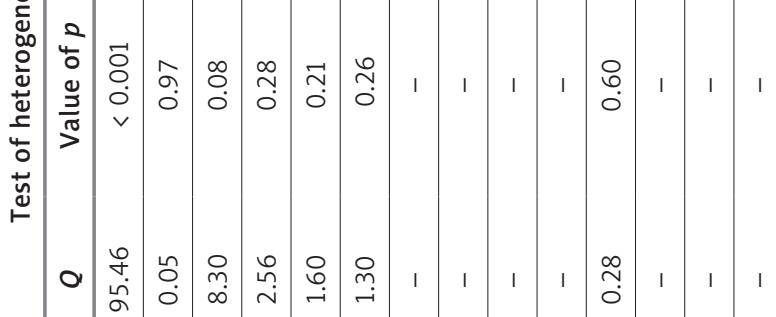

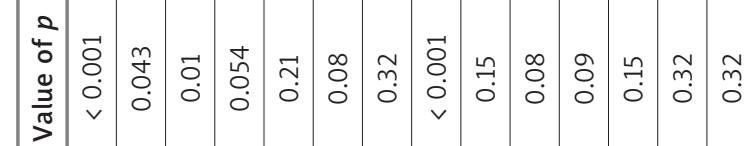

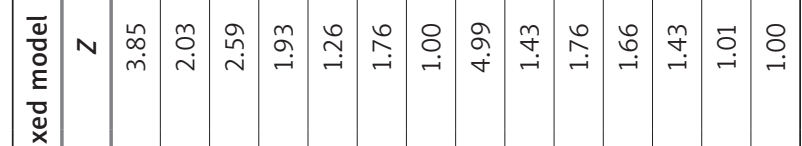

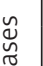

๘

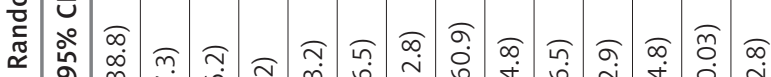

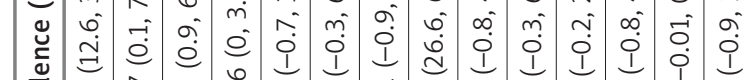

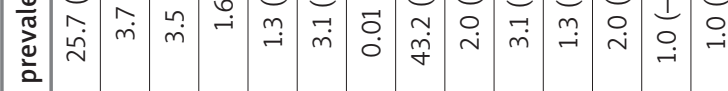
$\sum$

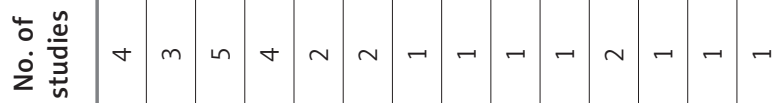

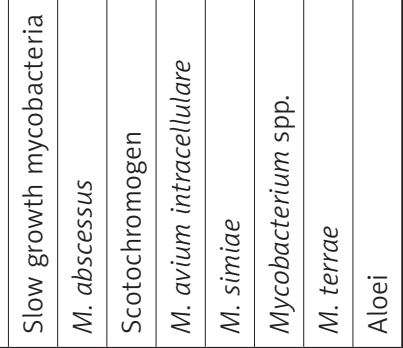


Moreover, the stratification of NTM species indicates that patients with bronchiectasis are susceptible to be infected with MAC, M. chelonae, $M$. fortuitum and $M$. abscessus, and long-term bacteria colonization is established. Our study supports the previous report that MAC bacteria were most frequently isolated (13-81\%) and were the most common cause of pulmonary NTM disease (43-81\%) [30]. Also, rapidly growing mycobacteria (RGM) (e.g. M. fortuitum complex, M. abscessus, $M$. chelonae) were frequently identified in pulmonary samples from Taiwan, China and Singapore [31-36]. Conversely, the species profile of NTM in patients without bronchiectasis is extremely different, with M. kansasii and M. xenopi predominating but MAC present in less than $10 \%$ of cases $[37,38]$. The mode of transmission of NTM is not well defined, but environmental exposure may be the major factor [39]. NTM existed in the outside environment, and were conditional pathogenic bacteria. Treatment of pulmonary NTM infection is complex, requiring multiple antibiotics and a prolonged treatment course [40]. With the application of antibiotics, genetic diseases such as cystic fibrosis of the lung, immunodeficiency disease and disorders of cilia movement were becoming the general causes of bronchiectasis [41]. NTM infections and $\alpha_{1}$-antitrypsin deficiency syndrome were all predisposing factors [42] which led to bronchiectasis becoming one of the potential factors in AIDS.

Some limitations of this study should be discussed. First of all, only published studies were included in the present meta-analysis. Thus, publication bias may exist, although the statistical test did not detect it. Secondly, significant heterogeneity was detected in the current meta-analysis. However, it was not a major problem because we did subgroup analysis to reduce the heterogeneity. Third, different populations may result in significant heterogeneity across the 8 eligible studies. Fourth, the symptoms or complications of NTM infected patients with bronchiectasis were not clearly reported. Fifth, the accuracy of TNM prevalence can be overestimated if the definite case only indicates patients with more severe symptoms which require a clinic visit. Finally, the present study was restricted to non-RCT trials, and a small number of selected trials. Thus, a larger number and higher quality of RCT studies are still warranted.

In conclusion, the present meta-analysis confirms the concept that the prevalence of NTM infection is high in patients with bronchiectasis. Additionally, it has identified that patients with bronchiectasis are susceptible to infection with long-term bacteria such as MAC, $M$. chelonae, M. fortuitum and M. abscessus. However, the sur- veillance of NTM species is relatively inaccurate for estimating the incidence of pulmonary disease and disease caused by MAC. Future studies should evaluate outcomes including the impacts of new tests on clinical decision making and the cost-effectiveness of new tests for NTM-infected patients.

\section{Acknowledgments}

Haiqing Chu and Lan Zhao contributed equally to this article.

The project was supported by grant No. 2010118 from the Shanghai Municipal Health Bureau and grant No. 12ZR1426200 from the Natural Science Foundation of Shanghai City.

\section{References}

1. Pappalettera M, Aliberti S, Castellotti P, Ruvolo L, Giunta V, Blasi F. Bronchiectasis: an update. Clin Respir J 2009; 3: 126-34

2. Carpenter JW, Mashima TY, Rupiper DJ. Exotic animal formulary. Saunders 2012.

3. Wittram C, Weisbrod G. Mycobacterium avium complex lung disease in immunocompetent patients: radiography - CT correlation. Br J Radiol 2002; 75: 340-4.

4. Barker AF. Bronchiectasis. N Engl J Med 2002; 346: 1383-93.

5. Wolinsky E. Nontuberculous mycobacteria and associated diseases. Am Rev Respir Dis 1979; 119: 107-59.

6. Falkinham Jr. Epidemiology of infection by nontuberculous mycobacteria. Clin Microbiol Rev 1996; 9: 177-215.

7. Von Reyn CF, Marlow J, Arbeit R, Barber T, Falkinham J. Persistent colonisation of potable water as a source of Mycobacterium avium infection in AIDS. Lancet Oncol 1994; 343: 1137-41.

8. Lynch DA, Simone PM, Fox MA, Bucher BL, Heinig MJ. CT features of pulmonary Mycobacterium avium complex infection. J Comput Assist Tomogr 1995; 19: 353-60.

9. Levin DL. Radiology of pulmonary Mycobacterium avium-intracellulare complex. Clin Chest Med 2002; 23: 603-12.

10. Miki M. [The pathophysiological concept of nontuberculous mycobacterial diseases and pulmonary Mycobacterium avium complex disease]. Nihon Rinsho 2011; 69: 1438-43.

11. Olivier KN. Nontuberculous mycobacterial pulmonary disease. Curr Opin Pulm Med 1998; 4: 148-53.

12. Kim RD, Greenberg DE, Ehrmantraut ME, et al. Pulmonary nontuberculous mycobacterial disease: prospective study of a distinct preexisting syndrome. Am J Respir Crit Care Med 2008; 178: 1066-74.

13. Falkinham III JO. The changing pattern of nontuberculous mycobacterial disease. Can J Infect Dis Med Microbiol 2003; 14: 281-6.

14. Moher D, Cook DJ, Eastwood S, Olkin I, Rennie D, Stroup DF. Improving the quality of reports of meta-analyses of randomised controlled trials: the QUOROM statement. Lancet 1999; 354: 1896-900.

15. Deeks JJ, Altman DG, Bradburn MJ. Statistical methods for examining heterogeneity and combining results from several studies in meta-analysis. Systematic reviews in Health Care: Meta-Analysis in Context 2001; 285-312. 
16. Higgins JP, Thompson SG, Deeks JJ, Altman DG. Measuring inconsistency in meta-analyses. BMJ 2003; 327: 557-60.

17. Mantel N, Haenszel W. Statistical aspects of the analysis of data from retrospective studies of disease. I Natl Cancer Inst 1959; 22: 719-48.

18. DerSimonian R, Laird N. Meta-analysis in clinical trials. Control Clin Trials 1986; 7: 177-88.

19. Egger M, Davey Smith G, Schneider M, Minder C. Bias in meta-analysis detected by a simple, graphical test. BMJ 1997; 315: 629-34.

20. Lee JY, Song JW, Hong SB, et al. Prevalence of NTM pulmonary infection in the patients with bronchiectasis. Tuberc Respir Dis 2004; 57: 311-9.

21. Palwatwichai A, Chaoprasong C, Vattanathum A, Wongsa A, Jatakanon A. Clinical, laboratory findings and microbiologic characterization of bronchiectasis in Thai patients. Respirology 2002; 7: 63-6.

22. Koh WJ, Lee KS, Kwon OJ, Jeong YJ, Kwak SH, Kim TS Bilateral bronchiectasis and bronchiolitis at thin-section CT: diagnostic implications in nontuberculous mycobacterial pulmonary infection. Radiology 2005; 235: 282-8.

23. Fowler S, French J, Screaton N, et al. Nontuberculous mycobacteria in bronchiectasis: prevalence and patient characteristics. Eur Respir J Suppl 2006; 28: 1204-10.

24. Wang GS, Wang Z, Yang L, Lin SL, Wu JS. Thoracoscopic management for bronchiectasis with non-tuberculous mycobacterial infection. Chin Med J 2008; 121: 2539-43.

25. Wickremasinghe M, Ozerovitch L, Davies G, et al. Non-tuberculous mycobacteria in patients with bronchiectasis. Thorax 2005; 60: 1045-51.

26. Tabarsi P, Baghaei P, Farnia P, et al. Nontuberculous mycobacteria among patients who are suspected for multidrug-resistant tuberculosis-need for earlier identification of nontuberculosis mycobacteria. Am J Med Sci 2009; 337: 182-4.

27. King PT, Holdsworth SR, Freezer NJ, Villanueva E, Holmes PW. Microbiologic follow-up study in adult bronchiectasis. Respir Med 2007; 101: 1633-8.

28. Keaveny TM, Pinilla TP, Crawford RP, Kopperdahl DL, Lou A. Systematic and random errors in compression testing of trabecular bone. J Orthop Res 1997; 15: 101-10.

29. Olivier KN, Yankaskas JR, Knowles MR. Nontuberculous mycobacterial pulmonary disease in cystic fibrosis. In: Seminars in respiratory infections 1996; 272-84.

30. Simons S, van Ingen J, Hsueh PR, et al. Nontuberculous mycobacteria in respiratory tract infections, eastern Asia. Emerg Infect Dis 2011; 17: 343-9.

31. Weimin L, Guanglu J, Zhihui L, et al. Non-tuberculous mycobacteria in China. Scand J Infect Dis 2007; 39: 138-41.

32. Teo S, Lo K. Nontuberculous mycobacterial disease of the lungs in Singapore. Singapore Med J 1992; 33: 464-6.

33. Chien H, Yu M, Wu M, Lin T, Luh K. Comparison of the BACTEC MGIT 960 with Lowenstein-Jensen medium for recovery of mycobacteria from clinical specimens. Int J Tuberc Lung Dis 2000; 4: 866-70.

34. Ding L, Lai C, Lee L, Hsueh P. Disease caused by non-tuberculous mycobacteria in a university hospital in Taiwan, 1997-2003. Epidemiol Infect 2006; 134: 1060-7.

35. Shih JY, Hsueh PR, Lee LN, et al. Nontuberculous mycobacteria isolates: clinical significance and disease spectrum. J Formos Med Assoc 1997; 96: 621-7.

36. Shu CC, Lee CH, Wang JY, et al. Nontuberculous mycobacteria pulmonary infection in medical intensive care unit: the incidence, patient characteristics, and clinical significance. Intensive Care Med 2008; 34: 2194-201.
37. Griffith DE. Management of disease due to Mycobacterium kansasii. Clin Chest Med 2002; 23: 613-21.

38. Olivier K. Nontuberculous mycobacterial pulmonary disease. Curr Opin Pulm Med 1998; 4: 148-53.

39. Glassroth J. Pulmonary disease due to nontuberculous mycobacteria. CHEST J 2008; 133: 243-51.

40. Leber A, Marras TK. The cost of medical management of pulmonary nontuberculous mycobacterial disease in Ontario, Canada. Eur Respir J 2011; 37: 1158-65.

41. Barker AF, Bardana EJ Jr. Bronchiectasis: update of an orphan disease. Am Rev Respir Dis 1988; 137: 969-78.

42. Holmes A, Pelton S, Steinbach S, Luzzi G. HIV related bronchiectasis. Thorax 1995; 50: 1227-7. 